\title{
A hybrid Force Position Control for a Upper Limb Rehabilitation Robot of Series Mechanism
}

\author{
Yali Liu , Linhong Ji \\ Division of Intelligent and Biomechanical System, State Key Laboratory of Tribology, Tsinghua University, 100084, Beijing, China
}

\begin{abstract}
Interactive rehabilitation robot which has better interaction is one main method to improve the patients' motion performance. The rehabilitation robot developed by Tsinghua University UECM for patients having weakness with shoulder and elbow should be improved by increasing interactive parts to adapt to patients' state. This paper described one control strategy to increase the interaction between robot and patients. The hybrid force position control for the upper limb rehabilitation robot UECM was developed to improve the patients' voluntary motion in exercises by controlling the robot to provide external forces according to the patients' forces and motion velocity. The hybrid force position controlled robot will be used for stroke patients under different states since its interactive control method
\end{abstract}

\section{Introduction}

Stroke is a leading cause of permanent disability in adults, with symptoms such as spasticity, hemiplegia, pain as well as leading to other complications[1,2]. Approximately two thirds of patients who sustained a stroke experience impaired motor function of an upper extremity and require continuous long term medical care to reduce their physical impairment, leaving much stress on families and much pressure on patients themselves $[3,4]$. Only about $50 \%$ of cognitively intact and medically stable patients with initially reduced upper extremity motor function obtain dexterity in the course of the first three months [5].

Recently, high intensive therapeutic interventions, such as constraint induced movement therapy and taskrelevant repetitive practice of the affected limb, have been identified as key factors for motor therapy after stroke [6-8]. Robots have high potential in the high intensive therapeutic interventions for its good repeatability and accuracy [9-12]. Besides, robots are equipped with sensors such as force sensors, encoders as well as accelerometers to record force, position and velocity exerted by patients, which can be used as evaluation for patient's performance quantitatively and objectively [13-16]. Research into rehabilitation robots has grown rapidly and the number of therapy rehabilitation robots has expanded dramatically since the first rehabilitation robot was developed in 1993[17-20]. In order to improve the muscle force generation of the paretic limb and to keep the effective muscular effort at its best, the rehabilitation robots trend to be smart enough to adapt to each patient's state and different states of one patient $[12,21,22]$
Rehabilitation robots usually provide three different motion types for hemiplegia patients (passive exercises, active exercises and active-assisted exercises) [15]. The paretic limb is guided by the rehabilitation robots during continuous passive exercises, which is proved to be effective in maintaining joint flexibility and stability in early stage after stoke (within 3 weeks of onset)[23]. Patients who have recovered and been strong enough to exercise themselves will get benefits from the active exercises. In active-assisted exercises, robots will supply external forces to help patients to achieve the exercises that patients cannot complete by themselves. Active voluntary attempts are found to be beneficial for patients in chronic stroke [23]. Thus, the interactive rehabilitation robots providing more motion types for different patients or for one patients under different states as well as attracting patients are becoming a trend in robot research.

\section{Methods}

Upper Extremity Compound Movement (UECM) rehabilitation robot [24], which was developed for shoulder-elbow coordination training by Tsinghua University has been found not intelligent enough to adapt to patients under different states with its actual passive and active exercises. The active-assisted and activeresistant exercises which need patients' voluntary movement are important for patients in chronic stroke [25], which need the robot and the patient interact with each other. Since UECM rehabilitation robot is an end effector robot, which connects itself with patients only by the end point, one important parameter is the forces between the robot and the patient. Once the forces can be changed with patients' states, the robot can adapt to 
patients ideally and can also attract patients to take exercises voluntarily with the personified interaction. In order to make UECM rehabilitation robot intelligent enough to interact with patients, the forces supplied by the robot should be adjustable according to the forces between the robot and patients' hands. The mechanical parts of UECM rehabilitation robot are shown in Figure 1, UECM rehabilitation robot has three parts: upper arm, forearm and interactive parts. The interactive parts consist of two main parts: the handle to fix patients' hand and a two dimensional force sensor to measure the interactive forces between robot and patients. The data from the two dimensional force sensor can be used to calculate the current of the two separate motor to provide external forces to achieve the task like drawing a circle or straight line.

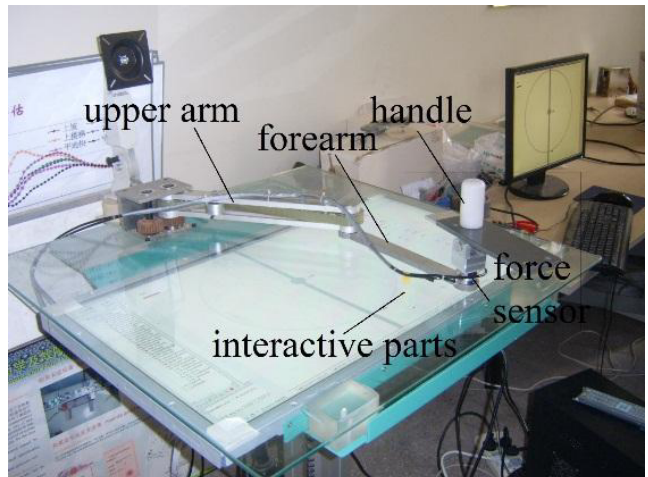

Figure 1. The mechanical parts of UECM rehabilitation robot

\subsection{Dynamic analysis of UECM rehabilitation robot}

The model can be described by Figure 2 .

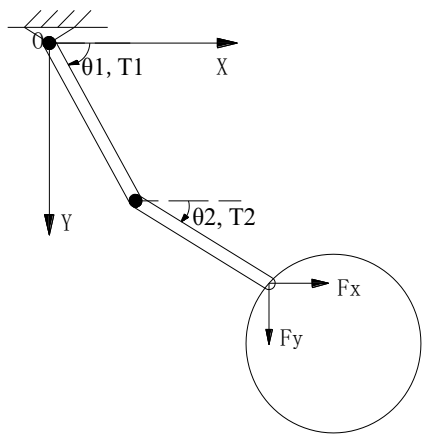

Figure 2. The mechanical model of UECM rehabilitation robot

In Figure 2, the circle refers to the task. $\boldsymbol{F} \boldsymbol{x}$ and $\boldsymbol{F} \boldsymbol{y}$ are the interactive forces between the robot and the patient, which are calculated by data of the two dimensional force sensor. $\boldsymbol{\theta}_{1}$ and $\boldsymbol{\theta}_{2}$ calculated according to the data of encoders installed on the motors of upper arm and forearm, are the angle between the arms and the horizontal line, which are used to describe the position of the rehabilitation robot end effector. $\boldsymbol{T} \boldsymbol{1}$ and $\boldsymbol{T} \boldsymbol{2}$ are calculated according to Lagrange's formulation.

$$
L=E_{k}+E_{p}
$$

$$
\begin{gathered}
=\frac{1}{2} m_{1} \boldsymbol{v}_{1}^{2}+\frac{1}{2} m_{2} \boldsymbol{v}_{2}^{2}+\frac{1}{2} J_{1} \omega_{1}^{2}+\frac{1}{2} J_{2} \omega_{2}^{2} \\
\boldsymbol{v}_{\mathbf{1}}=\left(-\frac{1}{2} l_{1} \dot{\boldsymbol{\theta}}_{1} \sin \boldsymbol{\theta}_{1} \quad \frac{1}{2} l_{1} \dot{\boldsymbol{\theta}}_{1} \cos \boldsymbol{\theta}_{1}\right)\left(\begin{array}{c}
\boldsymbol{i} \\
\boldsymbol{j}
\end{array}\right) \\
\boldsymbol{v}_{2}=\left(-l_{1} \dot{\boldsymbol{\theta}}_{1} \sin \boldsymbol{\theta}_{1}-\frac{1}{2} l_{2} \dot{\boldsymbol{\theta}}_{2} \sin \boldsymbol{\theta}_{2} l_{1} \dot{\boldsymbol{\theta}}_{1} \cos \boldsymbol{\theta}_{1}+\frac{1}{2} l_{2} \dot{\boldsymbol{\theta}}_{2} \cos \boldsymbol{\theta}_{2}\right)\left(\begin{array}{l}
\boldsymbol{i} \\
\boldsymbol{j}
\end{array}\right) \\
\boldsymbol{w}_{1}=\dot{\boldsymbol{\theta}}_{1} \\
\boldsymbol{w}_{2}=\dot{\boldsymbol{\theta}}_{2} .
\end{gathered}
$$

Since the motion task is in horizontal plane without height changes, $E_{p}=0$.

Then according to formulas (1-5)

$$
\begin{aligned}
& L=E_{k}+E_{p}=E_{k}=\left(\frac{1}{6} m_{1}+\frac{1}{2} m_{2}\right) l_{1}^{2} \dot{\boldsymbol{\theta}}_{1}^{2} \\
& +\frac{1}{6} m_{2} l_{2}^{2} \dot{\boldsymbol{\theta}}_{2}^{2}+\frac{1}{2} m_{2} l_{1} l_{2} \dot{\boldsymbol{\theta}}_{1} \dot{\boldsymbol{\theta}}_{2} \cos \left(\boldsymbol{\theta}_{1}-\boldsymbol{\theta}_{2}\right)
\end{aligned}
$$

According to Lagrange's formulation,

$$
\begin{aligned}
& \frac{d}{d t}\left(\frac{\partial \boldsymbol{L}}{\partial \boldsymbol{O}_{1}}\right)-\frac{\partial \boldsymbol{L}}{\partial \boldsymbol{O}_{1}}+F_{y} l_{1} \cos \boldsymbol{O}_{1}-F_{x} l_{1} \sin \boldsymbol{O}_{1}=T_{1} \\
& \frac{d}{d t}\left(\frac{\partial \boldsymbol{L}}{\partial \boldsymbol{O}_{2}}\right)-\frac{\partial \boldsymbol{L}}{\partial \boldsymbol{O}_{2}}+F_{y} l_{2} \cos \theta_{2}-F_{x} l_{2} \sin \theta_{2}=T_{2}
\end{aligned}
$$

According to formulas (6-8)

$$
\begin{aligned}
& \boldsymbol{T}_{1}=\left(\frac{1}{3} m_{1}+m_{2}\right) l_{1}^{2} \ddot{\boldsymbol{\theta}}_{1}+\frac{1}{2} m_{2} l_{1} l_{2} \ddot{\boldsymbol{\theta}}_{2} \cos \left(\boldsymbol{\theta}_{1}-\boldsymbol{\theta}_{2}\right) \\
& +\frac{1}{2} m_{2} l_{1} l_{2} \sin \left(\boldsymbol{\theta}_{1}-\boldsymbol{\theta}_{2}\right) \dot{\boldsymbol{\theta}}_{2}^{2}+\boldsymbol{F}_{\boldsymbol{y}} l_{1} \cos \boldsymbol{\theta}_{1}-\boldsymbol{F}_{\boldsymbol{x}} l_{1} \sin \boldsymbol{\theta}_{1} \\
& \boldsymbol{T}_{2}=\frac{1}{3} m_{2} l_{2}^{2} \ddot{\boldsymbol{\theta}}_{2}+\frac{1}{2} m_{2} l_{1} l_{2} \ddot{\boldsymbol{\theta}}_{1} \cos \left(\boldsymbol{\theta}_{1}-\boldsymbol{\theta}_{2}\right) \\
& -\frac{1}{2} m_{2} l_{1} l_{2} \sin \left(\boldsymbol{\theta}_{1}-\boldsymbol{\theta}_{2}\right) \dot{\boldsymbol{\theta}}_{1}^{2}+\boldsymbol{F}_{\boldsymbol{y}} l_{2} \cos \boldsymbol{\theta}_{2}-\boldsymbol{F}_{\boldsymbol{x}} l_{2} \sin \boldsymbol{\theta}_{2} \\
& \text { In the formulas }(9,10),
\end{aligned}
$$

$$
\begin{gathered}
F x=-F \text { sensor.x } \\
F y=-F \text { sensor.y }
\end{gathered}
$$

The Fsensor.x Fsensor.y sands for the forces data measured by the two dimensional sensor. The forces performed by robot and patient are used to keep the handle moving along the straight line or circle and other trajectory.

\subsection{Kinematic analysis of UECM rehabilitation robot}

In Figure 1, the aim is to keep the end point of robot to follow the circle. The kinematic formulas can be described following:

$$
\begin{aligned}
& \mathrm{x}=l_{1} \cos \theta_{1}+l_{2} \cos \theta_{2} \\
& \mathrm{y}=l_{1} \sin \theta_{1}+l_{2} \sin \theta_{2}
\end{aligned}
$$

According to formulas $(13,14)$, the Jacobi of the series mechanical system is calculated:

$$
\boldsymbol{J}=\left(\begin{array}{cc}
l_{1} \cos \theta_{1} & l_{2} \cos \theta_{2} \\
-l_{1} \sin \theta_{1} & -l_{2} \sin \theta_{2}
\end{array}\right)
$$




\subsection{Control model of the system}

The purpose of active-assisted and active-resistant motion types is to provide appropriate assistance or resistance for patients to perform their best to achieve the task. The two dimensional force sensor becomes the key to detect the state of patients, measuring the forces between the robot and the hand. According to the force data, the robot calculates the external forces according to formulas (912).

The control model of the system uses the hybrid force position control, which represents the calculated preset force value is related to the position information. The relationship between the position and forces can be described by the damper and spring, the formula (16).

$$
F_{\text {set }}=B \Delta \mathbf{v}+K \Delta \mathbf{x}
$$

In the formula $16, \Delta \mathbf{v}$ equals the error between the actual velocity and the preset velocity, $\Delta \mathbf{x}$ refers the error between the actual position and the preset position. The preset position means the task trajectory (a straight line or a circle). The control model of the interactive system is described by Figure 3.

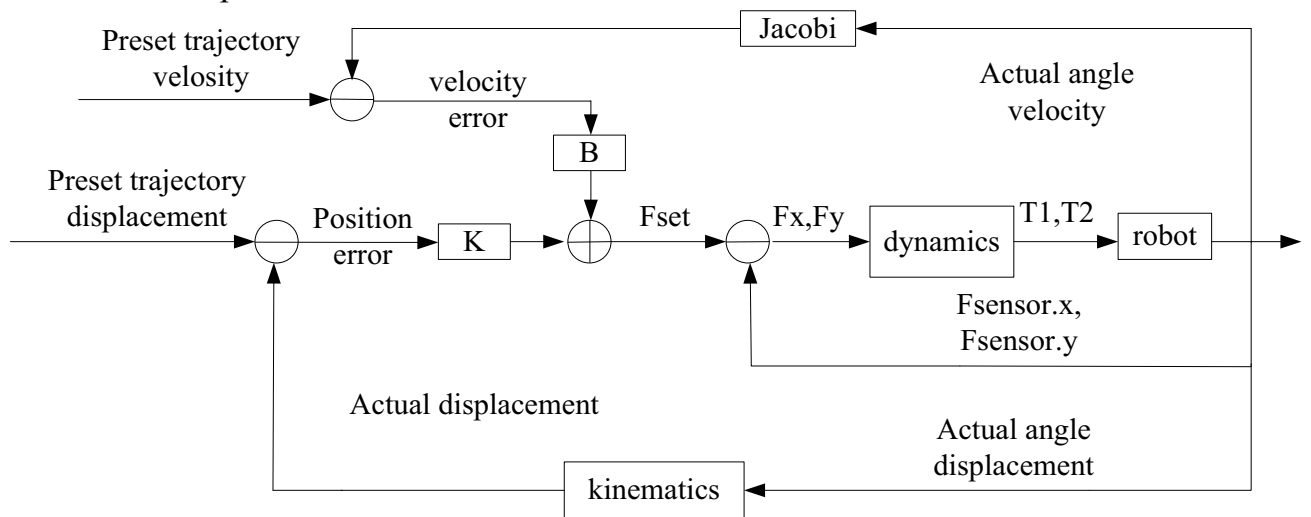

Figure 3. The hybrid force position control for UECM rehabilitation robot

The hybrid force position control changes the external forces provided by the robot according to the actual position to keep the motion direction along the task. The external forces $\mathrm{T} 1$ and $\mathrm{T} 2$ are calculated according to formulas $(9,10)$. In the exercises, safety is a main issue during the robot control, so the robot also calculates the power to judge whether the patient is in a spasm. If the calculated T1 or T2 is over the threshold, which is evaluated according to the patient' abilities in stretching their arms, the robot will stop providing the calculated forces to avoid making secondary damages to the patient.

\subsection{Strategy of control in tasks}

The rehabilitation exercises are used to improve the patients' movement performance by compulsorily intervention therapies. The robot keeps the patients moving their hands along the task trajectory to stretching their shoulder and elbow. In active-assisted and activeresistant motion types, the patients' voluntary movement cannot be along the trajectory accurately for their weakness, thus providing a motion band is an appropriate method for a better training. The objective of the robot is to keep patients moving the handle in the motion band and along the task trajectory.

The robot adjusts the forces in size and direction according to three different states during exercises. In the first state, while the handle in Fig 1 is in the motion band and the actual motion velocity is smaller than the threshold equaling the minimal velocity to perform a motion, the robot will provide external forces to assist the patient in the motion direction. In the second state, while the handle is in the motion band but the actual motion velocity is bigger than the other threshold meaning the maximal velocity for safety for the patient, which implies the patient is strong enough to perform against resistance, the robot will perform external forces to resist the patient in motion direction, namely provide the forces in the opposite direction of the trajectory. In the third state, while the handle is out of the motion band, the robot should supply the external forces in the direction that will drive the handle into the motion band along the vertical direction of the task trajectory direction. During the exercises, the external forces is calculated according to formula 16. Besides, the forces provided by the robot must be in the safety band for patient, namely the forces should be under the threshold that may make secondary damages to patients. The distribution of forces on the motion plane is described in Figure 4.

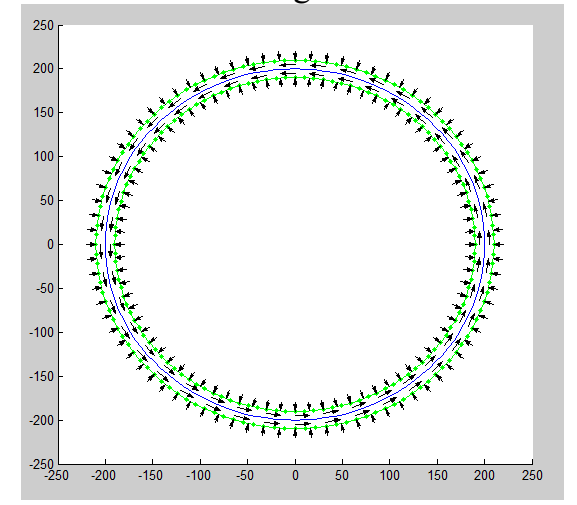


Figure 4. The distribution of forces provided by the robot

In Figure 4, the arrow stands for the force direction in the region. The green circles mean the maximal and minimal deviation from the task trajectory (the blue circle). The band between the maximal and minimal circle is the acceptable motion region. Out of the region, the robot will provide the external forces along the vertical direction pointing to the task trajectory. While in the region, the supplied forces are along the task trajectory direction or against the trajectory direction according to the motion velocity.

\section{Conclusion}

Rehabilitation robots are a main intervention therapy for its high intensity and its objective evaluation of patients' performance. The hybrid force position controlled UECM rehabilitation robot has better interaction for hemiplegic patients with weakness in upper limb. The hybrid force position controlled UECM rehabilitation robot adapts to patients' state by adjusting its provided external forces to patients according to the patients' interactive forces measured by a two dimensional force sensor, making it possible to train patients under different states. Patients will have a better feeling while exercising with the interactive robot than exercising with the stiff robot. The interactive rehabilitation robots will become a focus in rehabilitation robot research for its adaption to different states and to attract patients' voluntary motion.

\section{References}

1. X. Hu, K. Y. Tong, R. Song, V. S. Tsang, P. O. Leung, L. Li. Arch Phys Med Rehabil, 88, 10221029 (2007).

2. A. Basteris, S. M. Nijenhuis, A. H. Stienen, J. H. Buurke, G. B. Prange, F. Amirabdollahian, Journal of Neuroengineering \& Rehabilitation, 11, 19141926 (2014).

3. I. Brunner, J. S. Skouen, H. k. Hofstad, L. I. Strand, F. Becker, A. M. Sanders, et al. Bmc Neurology, 14, 1-5 (2014).

4. P. W. Duncan, L. B. Goldstein, D. Matchar, G. W. Divine, J. Feussner, Stroke, 23, 1084-1089 (1992).

5. G. Kwakkel, B. Kollen, Restorative Neurology \& Neuroscience, 25, 453-460 (2007).

6. C. Zollinger, Stroke, 24, 586-592 (1999).

7. G. G. Fluet, A. S. Merians, Q. Qiu, A. Davidow, S. V. Adamovich, Journal of Neuroengineering \& Rehabilitation, 11, 1440-1457 (2014).

8. M. Mihelj, T. Nef, R. Riener, Robotics and Automation 2007 IEEE International Conference on, 4120-4125 (2007).

9. J. A. Cozens, IEEE transactions on rehabilitation engineering: a publication of the IEEE Engineering in Medicine and Biology Society, 7, 254-256 (1999).

10. T. Nef, M. Mihelj, G. Colombo, R. Riener, Proceedings 2006 IEEE International Conference on, 3152 - 3157 (2006).
11. L. Dipietro, M. Ferraro, J. J. Palazzolo, H. I. Krebs, B. T. Volpe, N. Hogan, Neural Systems \& Rehabilitation Engineering IEEE Transactions on, 13, 325-334 (2005).

12. A. A. Blank, J. A. French, A. U. Pehlivan, M. K. O'Malley, Current Physical Medicine \& Rehabilitation Reports, 2, 184-195 (2014).

13. V. D. Perre, L. Sevit, R. Karsmakers, P. Peeraer, Louis, Congress of the European Society of Biomechanics, 19, 25-28 (2013).

14. S. J. Page, V. Hill, S. White, Clinical Rehabilitation, 27, 494-503 (2012).

15. G. B. Prange, M. J. Jannink, C. G. GroothuisOudshoorn, H. J. Hermens, M. J. Ijzerman, Journal of Rehabilitation Research \& Development, 43, 171183 (2006).

16. C. Spalletti, S. Lai, M. Mainardi, A. Panarese, A. Ghionzoli, C. Alia, Neurorehabilitation \& Neural Repair, 28, 188-196 (2014).

17. W. H. Chang Y. H. Kim, Journal of Stroke, 15, 174181 (2013).

18. P. Poli, G. Morone, G. Rosati, S. Masiero, Biomed Research International, 2013, 153872-153872 (2013).

19. N. Jarrassé, T. Proietti, V. Crocher, J. Robertson, A. Sahbani, G. Morel, Frontiers in Human Neuroscience, 8, 947-947 (2014).

20. R. Song, IEEE Engineering in Medicine and Biology Society. Conference, 4945-4948 (2006).

21. L. Brewer, F. Horgan, A. Hickey, D. Williams, Journal of the Association of Physicians, 106, 11-25 (2013).

22. F. Yakub, A. Z. Md Khudzari, Y. Mori, International Journal of Rehabilitation Research, 37, 9-21 (2014).

23. B. T. Volpe, M. Ferraro, D. Lynch, P. Christos, J. Krol, C. Trudell, Current Atherosclerosis Reports, 5, 314-319 (2005).

24. H. U. Yu-Chuan, J. I. Lin-Hong, Machinery Design \& Manufacture (2004).

25. S. E. Fasoli, H. I. Krebs, J. Stein, W. R. Frontera, N. Hogan, Archives of Physical Medicine \& Rehabilitation, 84, 477 - 482 (2003). 